\title{
Inhibition of $\beta$-catenin signaling protects against CTGF-induced alveolar and vascular pathology in neonatal mouse lung
}

\author{
Min Rong' ', Shaoyi Chen', Ronald Zambrano', Matthew R. Duncan², Gary Grotendorst ${ }^{2}$ and Shu Wu'
}

BACKGROUND: Bronchopulmonary dysplasia (BPD) is the most common and serious chronic lung disease of premature infants. Connective tissue growth factor (CTGF) plays an important role in tissue development and remodeling. We have previously shown that targeted overexpression of CTGF in alveolar type II epithelial cells results in BPD-like pathology and activates $\beta$-catenin in neonatal mice.

METHODS: Utilizing this transgenic mouse model and ICG001, a specific pharmacological inhibitor of $\beta$-catenin, we tested the hypothesis that $\beta$-catenin signaling mediates the effects of CTGF in the neonatal lung. Newborn CTGF mice and control littermates received ICG001 $(10 \mathrm{mg} / \mathrm{kg} / \mathrm{dose})$ or placebo (dimethyl sulfoxide, equal volume) by daily i.p. injection from postnatal day 5 to 15. Alveolarization, vascular development, and pulmonary hypertension (PH) were analyzed.

RESULTS: Administration of ICG001 significantly downregulated expression of cyclin D1, collagen 1a1, and fibronectin, which are the known target genes of $\beta$-catenin signaling in CTGF lungs. Inhibition of $\beta$-catenin signaling improved alveolar and vascular development and decreased pulmonary vascular remodeling. More importantly, the improved vascular development and vascular remodeling led to a decrease in $\mathrm{PH}$. CONCLUSION: $\beta$-Catenin signaling mediates the autocrine and paracrine effects of CTGF in the neonatal lung. Inhibition of CTGF- $\beta$-catenin signaling may provide a novel therapy for BPD.

\section{INTRODUCTION}

Bronchopulmonary dysplasia (BPD) is the most common and serious chronic lung disease of premature infants (1). Over the past four decades, the incidence of this disease has significantly increased as a result of improved survival of extremely-low-birthweight infants (2). The pathology of BPD is increasingly being recognized as a developmental arrest of the immature lung caused by injurious stimuli such as mechanical ventilation, oxygen exposure, and intrauterine or postnatal infections. Larger and simplified alveoli, decreased vascular growth, and variable interstitial fibrosis are the key pathological features observed in lungs of infants who died of BPD (1). The combination of decreased vascular growth and excessive pulmonary vascular remodeling leads to pulmonary hypertension $(\mathrm{PH})$ which significantly contributes to the morbidity and mortality of these infants (3). Yet, the underlying cellular and molecular mechanisms are poorly defined, and there is no effective therapy.

Connective tissue growth factor (CTGF) is a matricellular protein that plays an important role in tissue development and remodeling (4). Recent studies have highlighted the potential role of CTGF in BPD development and progression. The clinical association of CTGF with BPD is best established by studies demonstrating increased CTGF concentrations in bronchoalveolar lavage fluid from preterm infants developing BPD (5) and increased CTGF expression in lung tissues of infants who died of BPD (6). Multiple studies have examined the potential role of CTGF in experimental BPD and demonstrated that increased CTGF expression is associated with chronic hyperoxia as well as mechanical ventilationinduced lung injury in neonatal rodents (6-10). Recent studies from our laboratory utilizing genetic gain-of-function and biological loss-of-function approaches have provided compelling evidence that a novel signaling network orchestrated by CTGF plays an important role in BPD pathogenesis $(6,11,12)$. In our novel transgenic mouse model, targeted overexpression of CTGF in alveolar type II epithelial (AT II) cells induces the pathological hallmarks of severe BPD, including decreased alveolar and vascular development and increased vascular remodeling and PH (12). In contrast, in a hyperoxia-induced rat model of BPD, administration of a CTGF-neutralizing antibody improved alveolar and vascular development and decreased pulmonary vascular remodeling and $\mathrm{PH}$ (6). Interestingly, we have also found that CTGFinduced lung pathology is associated with activation of $\beta$-catenin signaling, whereas treatment with CTGF antibody inhibits hyperoxia-activated $\beta$-catenin signaling. It is known that CTGF can activate $\beta$-catenin signaling by binding to cell surface integrins and lipoprotein receptor-related protein (LRP) $5 / 6$, coreceptors of wingless/Int (Wnt) (13-16). It is unknown, however, whether activation of $\beta$-catenin signaling mediates CTGF-induced alveolar and vascular pathology in neonatal mice. 
The aim of this study was to test the hypothesis that activation of $\beta$-catenin signaling mediates CTGF-induced alveolar and vascular pathology in neonatal mice with targeted overexpression of CTGF in AT II cells. ICG001 is a newly developed small molecule that specifically inhibits $\beta$-catenin signaling by blocking $\beta$-catenin-forming transcription complex with lymphocyte enhancer factor (LEF)/T-cell factor (TCF) in the nuclei (17). ICG001 binds specifically to CBP but not the related transcriptional coactivator p300, thereby disrupting the interaction of CBP with $\beta$-catenin, and it has been shown to inhibit animal models of pulmonary fibrosis. We have previously shown that administration of ICG001 attenuates hyperoxia activation of $\beta$-catenin signaling and induction of alveolar damage in neonatal rats (18). We demonstrated here that treatment with ICG001 inhibits expression of $\beta$-catenin target genes, improves alveolar and vascular development, and decreases pulmonary vascular remodeling in neonatal mice with targeted overexpression of CTGF in AT II cells. These data indicate that activation of $\beta$-catenin signaling plays an important role in CTGF-induced BPD-like pathology, and targeting $\mathrm{CTGF} / \beta$-catenin signaling may provide a novel strategy for preventing and treating BPD in premature infants.

\section{RESULTS}

\section{Targeted Overexpression of CTGF in AT II Cells Increases CTGF Concentration in BAL and Serum}

Our previous studies have shown that high levels of CTGF expression are detected in AT II cells and lung homogenates in CTGF transgenic mice at $2 \mathrm{wk}$ of age, and this is in a sharp contrast to the control mice that have barely detectable CTGF expression in either AT II cells or lung homogenates (12). Since CTGF is a secretory protein, we hypothesized that AT II cells would be able to secrete CTGF into the alveolar airspaces as well as the blood stream. We therefore assessed CTGF concentrations in BAL and serum by ELISA in 4-wk-old mice, given the small sizes of mice and difficulty in performing BAL and collecting blood at $2 \mathrm{wk}$ of age. The CTGF mice had high levels of CTGF, whereas control mice had extremely low level of CTGF in their BAL (Figure 1). CTGF mice also had increased CTGF concentration in their serum as compared with control mice (Figure 1). These results suggested that CTGF can be secreted by AT II cells into alveolar airspaces and blood stream in CTGF transgenic mice, thus eliciting both autocrine and paracrine effects on the lung and possible extra-pulmonary organs.

\section{ICG-001 Inhibits CTGF Activation of $\beta$-Catenin Signaling}

To evaluate the effectiveness of ICG001 in blocking CTGF induction of $\beta$-catenin signaling, we first examined $\beta$-catenin signaling by assessing $\beta$-catenin localization. There was an increased $\beta$-catenin nuclear translocation in CTGF transgenic lungs that were exposed to the placebo as compared with the control lungs (Figure 2a,b). There was no significant difference in the index of $\beta$-catenin nuclear translocation between placebo treatment and ICG001 treatment in CTGF mice (Figure 2a,b). We then assessed expression of cyclin D1,

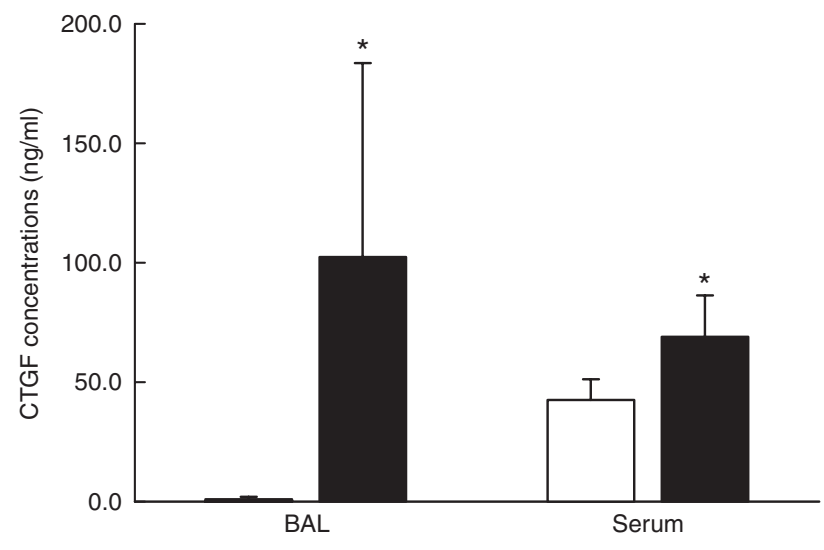

Figure 1. Targeted overexpression of CTGF in AT II cells increases CTGF concentrations in BAL and serum. CTGF concentration was measured by ELISA in the BAL and serum from control and CTGF transgenic mice at $4 \mathrm{wk}$ of age. There were significantly higher concentrations of CTGF detected in BAL and serum from CTGF transgenic mice. $n=8 /$ group. ${ }^{*} P<0.01 \mathrm{com}-$ pared to control mice. Open bar: control; Solid bar: CTGF + transgenes.

collagen 1a1, and fibronectin, known $\beta$-catenin target genes. As demonstrated in Figure 2c,d, targeted overexpression of CTGF in AT II cells increased cyclin D1 protein expression in the presence of the placebo. However, treatment with ICG001 drastically decreased cyclin D1 expression. There were increased expression of collagen 1a1 and fibronectin mRNAs in CTGF lungs that were treated with the placebo, but administration of ICG001 decreased expression of these genes to nearcontrol levels (Figure 2e,f). Thus, ICG001 effectively blocks CTGF activation of $\beta$-catenin signaling.

\section{Inhibition of $\beta$-Catenin Signaling Improves Alveolarization in CTGF Mice}

On histological examination, the control lungs exposed to either placebo or ICG001 displayed normal alveolar development with numerous small alveoli and secondary septa (Figure 3a). In contrast, there were larger and simplified alveoli with fewer secondary septa in CTGF lungs that exposed to the placebo (Figure 3a). However, the CTGF lungs exposed to ICG001 presented with improved alveolar structure that appeared similar to the control lungs. Further morphometric analysis demonstrated that the placebo-treated CTGF lungs have increased mean linear intercept as compared to the control lungs (Figure 3b). But treatment with ICG001 significantly decreased mean linear intercept in CTGF lungs (Figure 3b). These data indicate that inhibition of $\beta$-catenin signaling improves alveolar development in neonatal mice with targeted overexpression of CTGF in AT II cells.

\section{Inhibition of $\beta$-Catenin Signaling Increases Vascular Development in CTGF Mice}

To determine the effects of ICG001 on lung vascular development, vascular density was quantified on vWF immunofluorescence-stained tissue sections. Compared to the control lungs, there was a near 50\% decrease in vWF-positive intraacinous vessels in CTGF lungs that were treated with the placebo 


\section{Articles | Rongetal.}

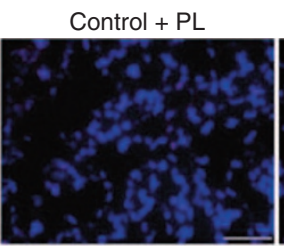

Control + ICG

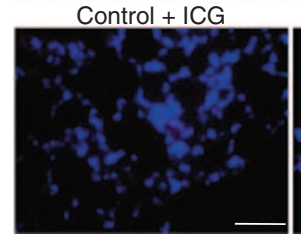

d

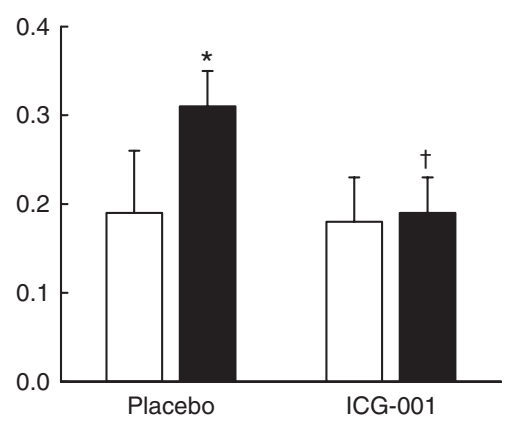

CTGF + ICG

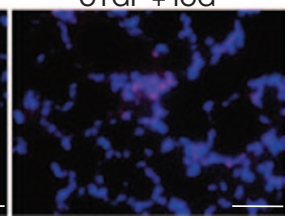

b

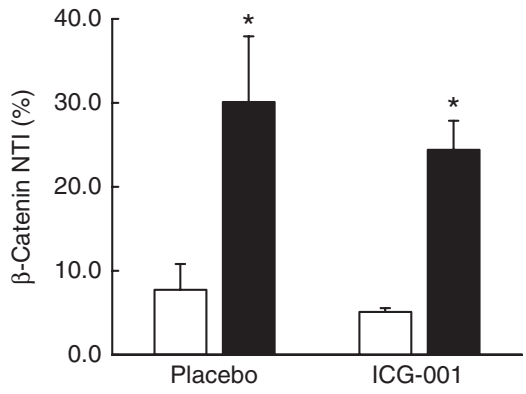

e

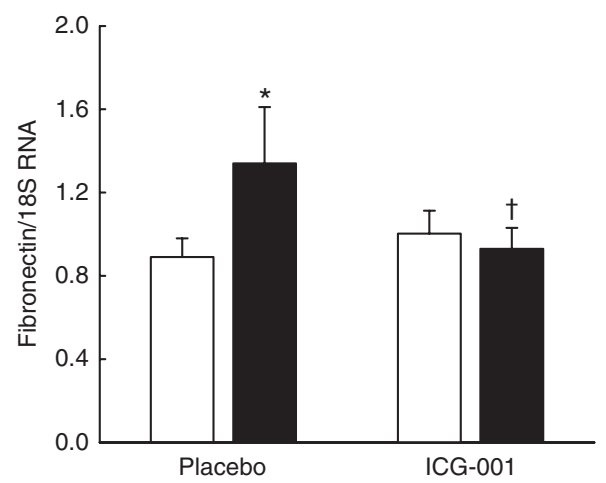

c

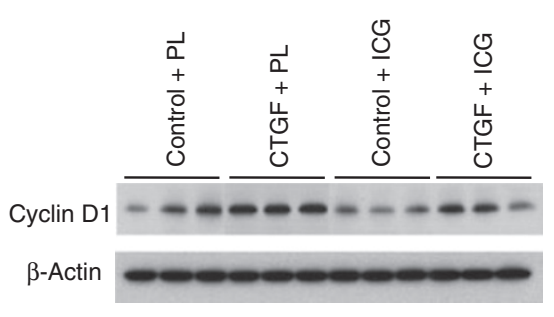

f

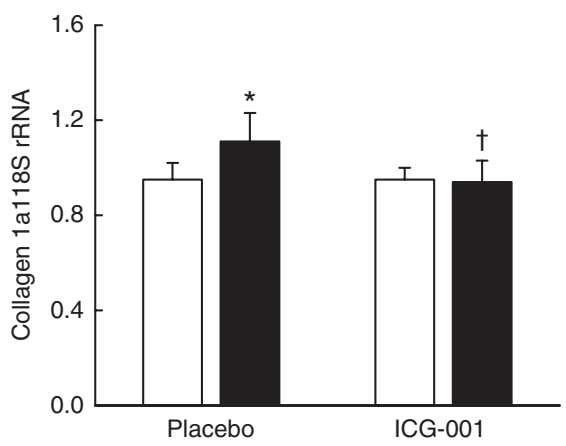

Figure 2. ICG001 inhibits $\beta$-catenin signaling. (a) Immunofluorescence staining for $\beta$-catenin (red signal) and DAPI nuclear staining (blue signal) were performed. Pink signals (white arrows) indicate colocalization of $\beta$-catenin with nuclei. Magnification: $\times 40 \mathrm{bar}=50 \mu \mathrm{m}$. (b) $\beta$-catenin nuclear translocation index (NTI) was determined by the percentage of $\beta$-catenin-positive nuclei/total nuclei. The CTGF transgenic mice had an increased $\beta$-catenin NTI compared with control mice, and this was not affected by administration of ICG001. $n=5 /$ group; ${ }^{*} P<0.001$ compared with control mice. (c) Representative western blot photographs. ICG, ICG001; PL, placebo. (d) Densitometry analysis demonstrated that CTGF transgenic mice have an increased cyclin D1 expression in the presence of the placebo, and this was significantly decreased by ICG001 treatment. $n=6 /$ group; ${ }^{*} P<0.01$ compared with control groups; ${ }^{\text {t+ } P}<0.01$ compared with CTGF + placebo group. (e) Fibronectin gene expression was analyzed by qRT-PCR. CTGF transgenic mice had an increased fibronectin gene expression in the presence of the placebo. Treatment with ICG001 decreased fibronectin gene expression in CTGF mice. $n=5 /$ group; ${ }^{*} P<0.01$ compared with control mice; ${ }^{+} P<0.01$ compared with placebo-treated CTGF mice. (f) Collagen 1 a1 gene expression was increased in the placebo-treated CTGF transgenic mice, and this was decreased by exposure to ICG001. $n=5 /$ group; ${ }^{*} P<0.01$ compared with control mice; ${ }^{\dagger} P<0.05$ compared with placebo-treated CTGF mice. Open bar: control; Solid bar: CTGF + transgenes.

(Figure 4a,b). Whereas administration of ICG001 increased vascular density in the CTGF lungs (Figure 4a,b). These results indicate that $\beta$-catenin signaling mediates CTGF inhibition of lung vascular development.

\section{Inhibition of $\beta$-Catenin Signaling Decreases Pulmonary Vascular Remodeling in CTGF Mice}

To determine the effects of ICG001 on pulmonary vascular remodeling, we performed immunostaining for $\alpha$-SMA, a smooth muscle marker, and assessed medial wall thickness (MWT). The peripheral vessels in the placebo-treated CTGF lungs displayed increased $\alpha$-SMA expression as compared with the control lungs and ICG001-treated CTGF lungs (Figure 5a). Measurements of MWT was increased near twofold in the placebo-treated CTGF lungs, and this was significantly decreased by treatment with ICG001 (Figure 5b). To answer the question whether this increased MWT is related to increased vascular smooth cell proliferation, we performed double immunofluorescence with $\alpha$-SMA and Ki-67, a nuclear proliferating marker. As illustrated in Figure 5c,d, there were increased vessels that are positively stained for Ki-67 in CTGF lungs that received the placebo. Treatment with ICG001 decreased Ki-67 positively stained vessels in the CTGF lungs as compared with placebo treatment (Figure $5 \mathrm{c}$,d). Fibronectin is an important component of extracellular matrix (ECM) in the vasculature. Immunofluorescence staining showed abundant fibronectin expression in the walls of peripheral vessels $(<50$ $\mu \mathrm{m}$ in diameter) in the CTGF lungs that were exposed to the placebo, and this was decreased by ICG001 (Figure 5e). These data indicate that epithelial overexpression of CTGF results in excessive pulmonary vascular remodeling, and this is mediated by $\beta$-catenin signaling.

\section{Inhibition of $\beta$-Catenin Signaling Decreases PH in CTGF Mice}

To determine whether the increased vascular development and reduced pulmonary vascular remodeling leads to improvement of PH in CTGF mice, we studied right ventricular systolic pressure (RVSP) and right ventricular hypertrophy (RVH) by measuring $\mathrm{RV} / \mathrm{LV}+\mathrm{S}$ weight ratio. The CTGF mice had significantly higher RSVP and RVH as compared with the 

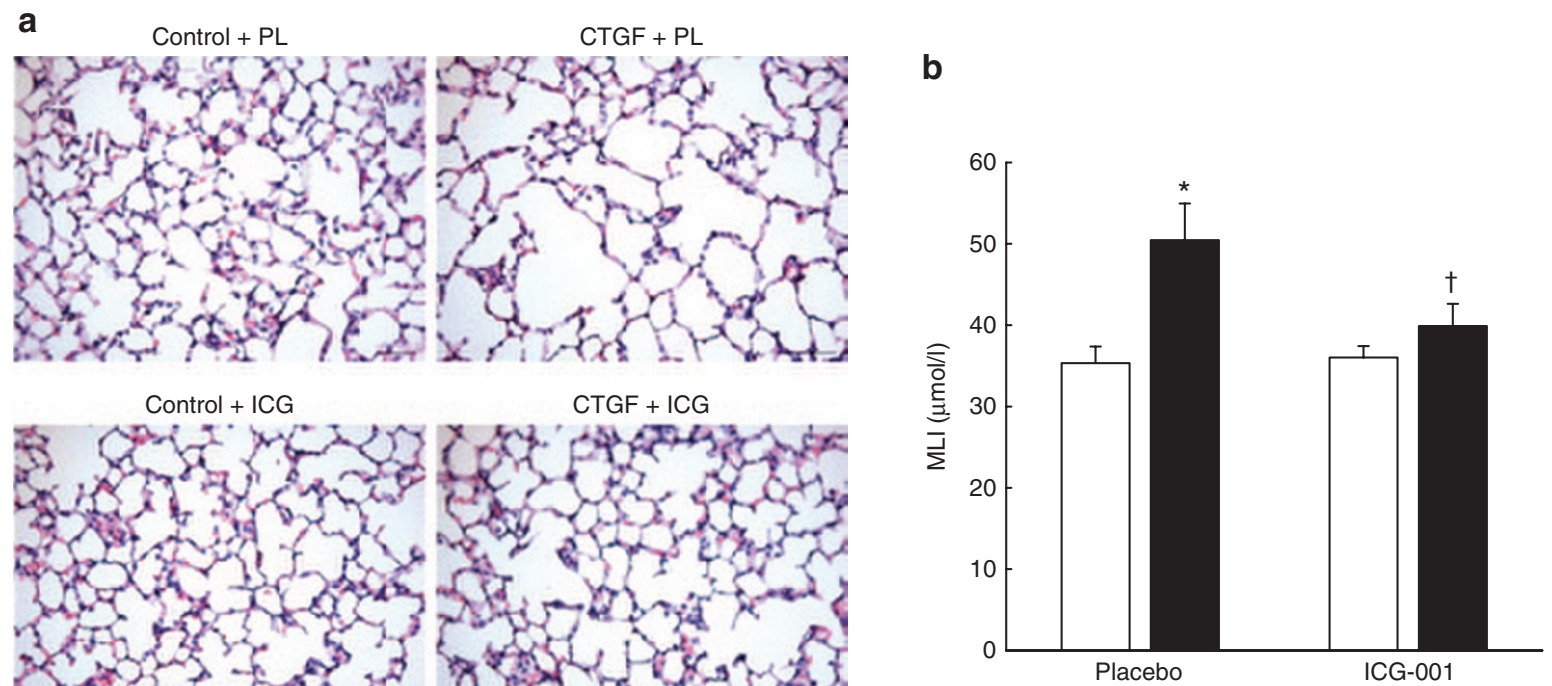

Figure 3. Inhibition of $\beta$-catenin improves alveolarization. (A) On histological examination, lungs from placebo-treated CTGF mice displayed an impairment of alveolarization with fewer, larger, and simplified alveoli. Treatment with ICG001 improved alveolar structures that appeared similar to the control lungs. Bar $=50 \mu \mathrm{m}$. (b) Lung morphometry measured by mean linear intercept (MLI) demonstrated that placebo-exposed CTGF mice have an increased MLI as compared with control mice. Administration of ICG001 decreased MLI in CTGF mice. $n=5 /$ group; ${ }^{*} P<0.001$ compared with control mice; ${ }^{\dagger} P<0.001$ compared with placebo-treated CTGF mice. Magnification: $\times 20$ and bar $=50 \mu \mathrm{m}$. Open bar: control; Solid bar: CTGF + transgenes.

a

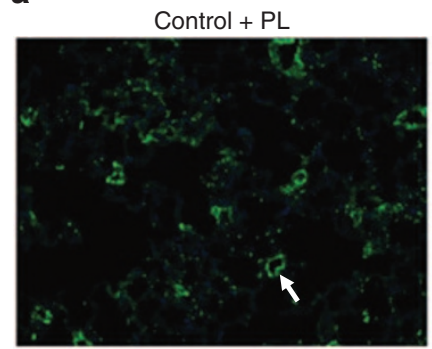

Control + ICG

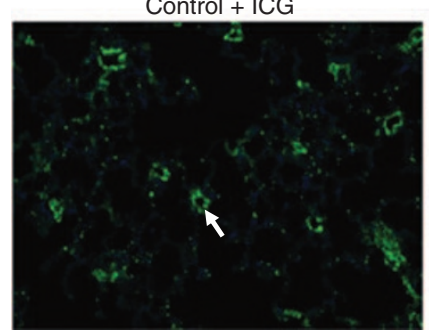

$\mathrm{CTGF}+\mathrm{ICG}$

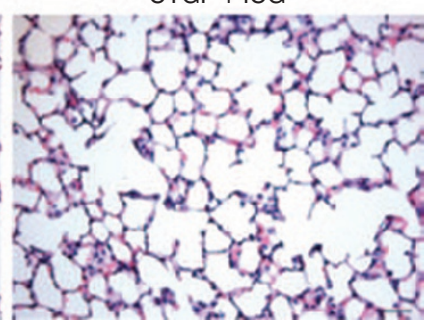

b

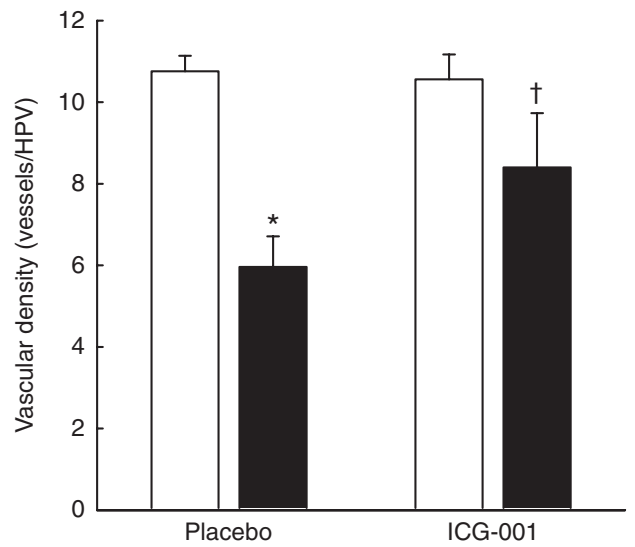

Figure 4. Inhibition of $\beta$-catenin improves vascular development. (a) Immunofluorescence staining for vWF (green signal). Bar $=50 \mu \mathrm{m}$. (b) Vascular density was determined by counting vWF-positive vessels $(<50 \mu \mathrm{m}$ in diameter) on five random high power view (HPV) images from each lung section. The vascular density was significantly lower in the placebo-treated CTGF lungs as compared with control lungs. Administration of ICG001 modestly increased vascular density in CTGF lungs. $n=5$ /group; ${ }^{*} P<0.001$ compared with control lungs; ${ }^{+} P<0.01$ compared with placebo-treated CTGF lungs. Magnification: $\times 20$ and bar $=50 \mu \mathrm{m}$. Open bar: control; Solid bar: CTGF + transgenes.

control mice (Figure 6). But treatment with ICG001 decreased both RVSP and RVH in CTGF mice. These results support that ICG001 decreases CTGF-induced PH in neonatal mice.

\section{DISCUSSION}

In this study, we provide evidence that $\beta$-catenin signaling mediates AT II cell-CTGF induced BPD-like pathology in neonatal mice. We demonstrated that administration of ICG001, a specific $\beta$-catenin inhibitor that directly blocks $\beta$-catenin transcriptional activity, effectively decreased CTGF-induced expression of $\beta$-catenin target genes that are known to be involved in lung injury and vascular remodeling. Furthermore, ICG001 treatment improved alveolar and vascular development and decreased pulmonary vascular remodeling and $\mathrm{PH}$ in CTGF transgenic mice. This study, therefore for the first time, highlights the functional significance of CTGF- $\beta$-catenin signaling in the development and progression of BPD-like pathology in a mouse model. 


\section{Articles | Ronget al.}

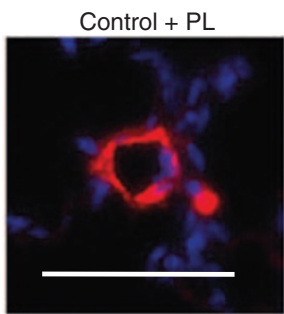

Control + ICG

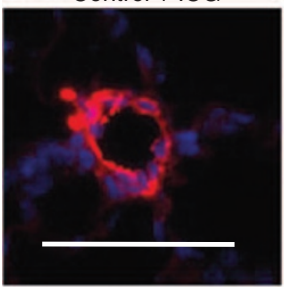

C

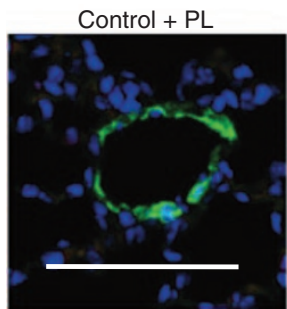

Control + ICG

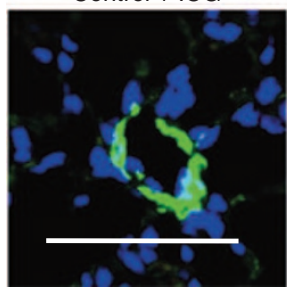

e

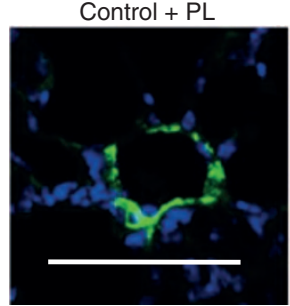

$\mathrm{CTGF}+\mathrm{PL}$

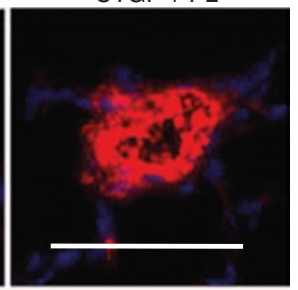

CTGF + ICG

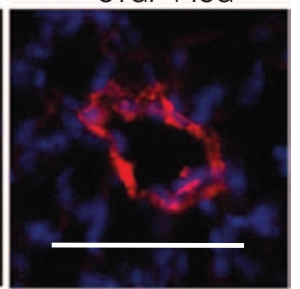

CTGF + PL

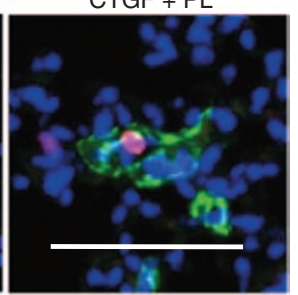

CTGF + ICG

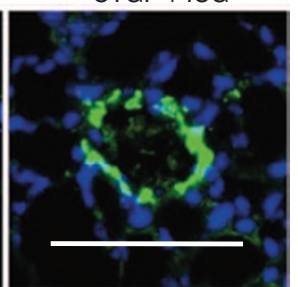

$\mathrm{CTGF}+\mathrm{PL}$

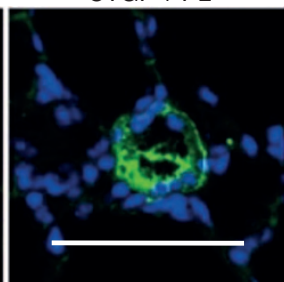

b

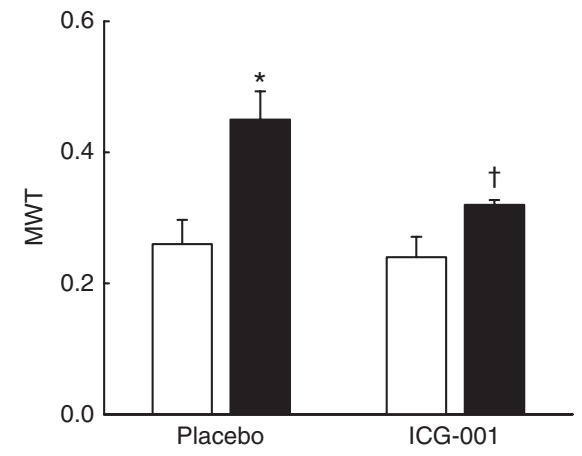

d

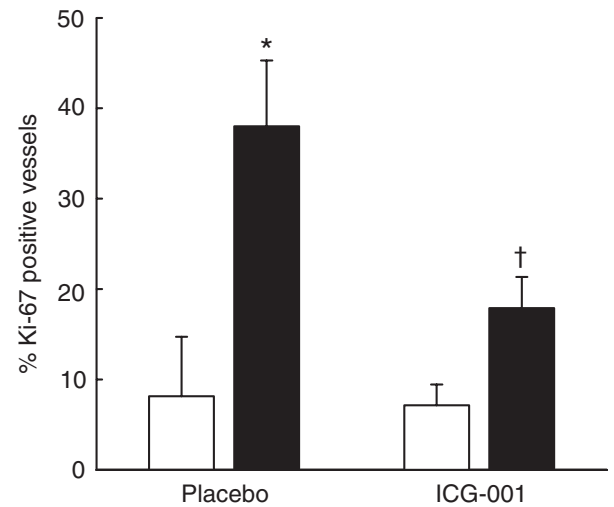

Control + ICG

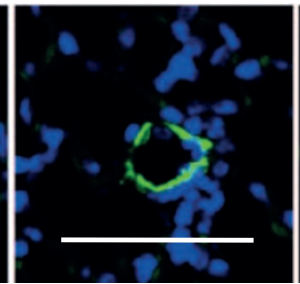

$C T G F+I C G$

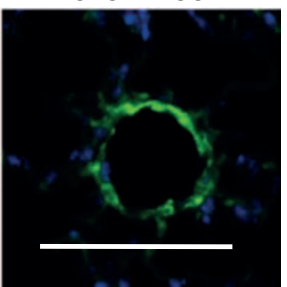

Figure 5. Inhibition of $\beta$-catenin decreases pulmonary vascular remodeling. (a) Immunofluorescence staining for $\alpha$-SMA (red signal) and DAPI nuclear staining (blue signal). ICG, ICG001; PL, placebo. Bar $=50 \mu \mathrm{m}$. (b) Medial wall thickness (MWT) was assessed from 20 peripheral vessels $(<50 \mu \mathrm{m}$ in diameter) on each lung section. CTGF mice had an increased MWT, but ICG001 administration decreased MWT in CTGF transgenic mice. $n=5 /$ group; ${ }^{*} P<0.001$ compared to control mice; ${ }^{\dagger} P<0.001$ compared with CTGF + placebo group. (c) Double immunofluorescence staining for $\alpha$-SMA (green signal), Ki67 (red signal), and DAPI nuclear staining (blue signal). ICG, ICG001; PL, placebo. Bar $=50 \mu \mathrm{m}$. (d) The percentage of vessels $(<50 \mu \mathrm{m}$ in diameter) with at least one positive $\mathrm{Ki}-67$ nucleus was significantly increased in CTGF lungs that were exposed to the placebo. Treatment with ICG001 decreased this index in CTGF lungs. $n=$ 5/group; ${ }^{*} P<0.001$ compared with the control groups; ${ }^{\dagger} P<0.001$ compared with CTGF + placebo groups. (e) Immunofluorescence staining for fibronectin (green signal) and DAPI nuclear staining (blue signal). Fibronectin staining was mainly detected in the vessel walls, and this appeared to be increased in the thickened vessels of CTGF + placebo lungs. ICG, ICG001; PL, placebo. Magnification: $\times 100$ and bar $=50 \mu \mathrm{m}$. Open bar: control; Solid bar: CTGF + transgenes.

Recent advances in genetic modification technology have provided us with the ability to highlight the cell-specific role of CTGF in lung development and pathology. Ivkovic et al. (19) have shown that global knockout of CTGF results in respiratory failure and immediate death in newborn mice suggesting that endogenous CTGF in lung cells are important for embryonic lung development and newborn lung function.
Sonnylal et al. $(20,21)$ demonstrated that targeted overexpression of CTGF in lung fibroblasts disrupts lung structure and induces fibrosis which affects both lung fibroblasts and epithelial cells. Our previous studies showed that targeted overexpression of CTGF in AT II cells not only disrupts alveolar and vascular development but also induces interstitial fibrosis and vascular remodeling (12). These data indicate that epithelial 


\section{CTGF, $\beta$-catenin, and neonatal lung}

a

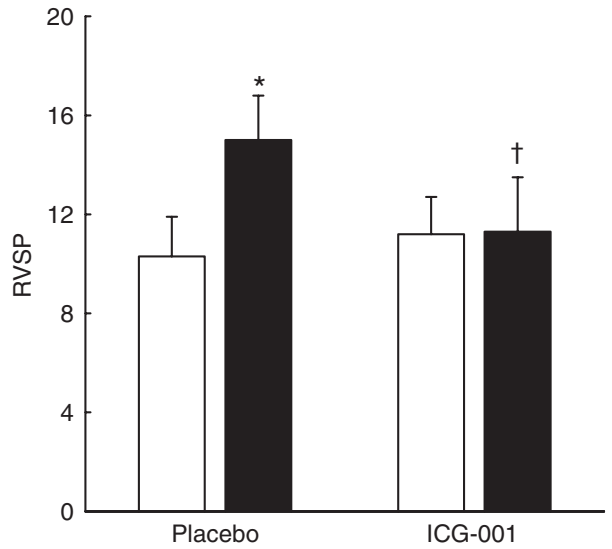

b

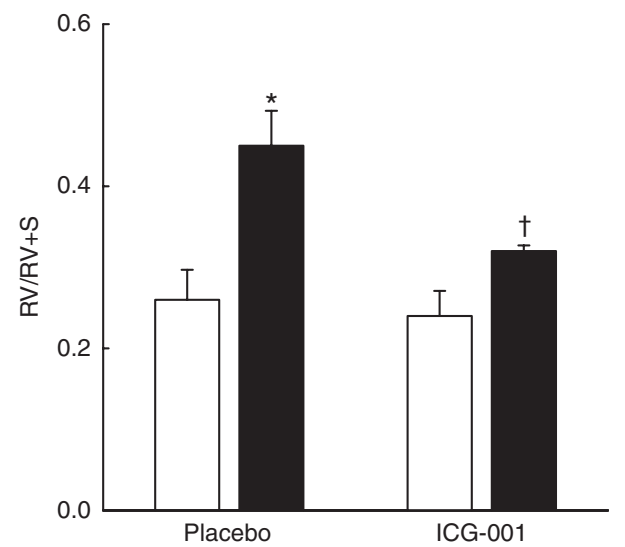

Figure 6. Inhibition of $\beta$-catenin decreases PH. (a) Right ventricular systolic pressure (RVSP) was significantly increased in the placebo-treated CTGF lungs as compared with CTGF + placebo group. ICG001 administration decreased RVSP to near control groups. $n=6 /$ group; ${ }^{*} P<0.01$ compared with the control mice; ${ }^{\dagger} P<0.05$ compared with CTGF + ICG001 group. (b) The weight ratio of right ventricle (RV) to left ventricle + septum (LV + S) was measured as an index for right ventricular hypertrophy (RVH). CTGF transgenic mice had an increased RVH, and this was decreased by ICG001 treatment. $n=6 /$ group; ${ }^{*} P<0.001$ compared with control mice; ${ }^{\dagger} P<0.001$ compared with CTGF + placebo mice. Open bar: control; Solid bar: CTGF + transgenes.

derived or fibroblast-derived CTGF is a critical inducer of BPD-like pathology.

We showed here for the first time that CTGF transgenic mice have elevated CTGF concentration in BAL and serum, indicating that CTGF can be secreted from AT II cells into alveolar airspaces and blood stream in neonatal mice. This finding raises the possibility that epithelial derived CTGF can directly induce BPD-like pathology by both autocrine and paracrine mechanisms. Furthermore, AT II cell-secreted CTGF can also have extra-pulmonary effects on other developing organs, particularly the heart, given that we have previously shown right ventricular hypertrophy in CTGF transgenic mice (12).

$\beta$-Catenin is a key regulatory protein with bidirectional capacity to tightly regulate nuclear transcription and affect cell migration and adhesion by closely interacting with the cytoskeleton and adherens junction cadherins (22). A multiprotein "destruction complex" continually phosphorylates $\beta$-catenin, flagging it for degradation by the ubiquitin/proteosome system. Inhibition of this phosphorylation by activation of Wnt and ILK signaling allows $\beta$-catenin to accumulate in the cytoplasm and enter the nucleus to form a transcriptional complex localized at gene promoters. $\beta$-Catenin recruits the transcription coactivators, cyclic AMP response-element binding protein binding protein $(\mathrm{CBP})$, or its closely related homolog, p300, as well as other components of the basal transcription machinery $(22,23)$. The transcription complex formed by $\beta$-catenin and its DNA-binding partners known as LEF/TCF is able to promote chromatin remodeling and transcriptional initiation in a cell type-specific and context-specific manner, thus playing a key role in embryonic development, cell proliferation, differentiation, and survival. CTGF can directly bind to cell surface integrin complexes thus activating ILK signaling $(13,14)$. CTGF can also directly bind to LRP5/6, coreceptors of Wnt $(15,16)$. Activation of ILK and LRP5/6 can lead to nuclear translocation of $\beta$-catenin and subsequent activation of target gene transcription. We and others have previously demonstrated that $\beta$-catenin signaling is increased in the lungs of hyperoxia-exposed neonatal rats, a widely used experimental model of BPD $(6,24)$. A recent study demonstrated that increased $\beta$-catenin is present in the mesenchymal stromal cells isolated from infants developing BPD and in $\alpha$-SMA-positive myofibroblasts in the lungs of infants dying of BPD (25). Importantly, we have recently shown that targeted overexpression of CTGF in AT II cells activates $\beta$-catenin signaling while antagonism of CTGF inhibits hyperoxia activation of $\beta$-catenin signaling in the neonatal lungs $(6,12)$. These data highlight that $\beta$-catenin signaling could be an important pathway that CTGF utilizes to induce lung pathology.

We extended our previous findings that targeted overexpression of CTGF in AT II cells activates $\beta$-catenin signaling by confirming that there were increased $\beta$-catenin nuclear translocation and expression of its three target genes including cyclin D1, collagen 1a1, and fibronectin in CTGF transgenic lungs. These three genes were chosen because they are known to be involved in lung injury and remodeling. Cyclin $\mathrm{D} 1$ is a cell cycle regulatory molecule that is active in G1 phase transition and functions as an important mitogenic regulator (26). Increased cyclin D1 expression is linked to acute and chronic lung injuries induced by inflammatory agents, and it is postulated to contribute to interstitial cell proliferation and fibrosis formation $(27,28)$. Collagen 1a1 and fibronectin are the main components of ECM that participate in tissue and vascular remodeling. Increased collagen $1 \mathrm{a} 1$ and fibronectin expression is consistently associated with pulmonary fibrosis $(29,30)$. We showed that treatment with ICG001 did not affect $\beta$-catenin nuclear translocation index but drastically downregulated cyclin D1, collagen I $\alpha 1$, and fibronectin expression in CTGF transgenic lungs. These results are consistent with our previous findings in hyperoxia-induced neonatal injury model in that ICG001 did not affect $\beta$-catenin nuclear transcription index but drastically downregulated cyclin D1 expression. 


\section{Articles Rongetal.}

Our previous studies have demonstrated that targeted overexpression of CTGF in AT II cells activates $\beta$-catenin signaling and disrupts alveolar epithelial cell homeostasis via an autocrine mechanism. In CTGF transgenic lungs, part of the $\beta$-catenin nuclear translocation was detected in AT II cells (31). Furthermore, overexpression of CTGF induces $\beta$-catenin nuclear translocation and disrupts alveolar-like cyst formation in cultured AT II cells (12,31). In this study, we showed that administration of a $\beta$-catenin inhibitor significantly improved alveolarization in CTGF transgenic lungs. Thus, the improved alveolar development in responding to $\beta$-catenin inhibition is at least in part mediated by an autocrine mechanism. Previous genetic loss-of-function and gain-of-function studies have highlighted an important role of $\beta$-catenin signal transduction pathway in cell fate specification, cell differentiation, and formation of alveolar structure during embryonic and postnatal lung development. Targeted deletion of $\beta$-catenin in developing respiratory epithelium disrupted AT II and AT I cell differentiation and alveolar formation in mice (32). Whereas targeted overexpression of an active form of $\beta$-catenin under the control of SP-C promotor caused ectopic differentiation of AT II like cells in conducting airways, globlet cell hyperplasia, and air space enlargement in the postnatal lung of mice (33). Our results identified a novel growth factor and transcription factor interaction, the CTGF- $\beta$-catenin signaling pathway that is crucial for regulating alveolarization in the postnatal lung.

Alveolar development and angiogenesis in the lung go hand in hand, and impairment of alveolarization has negative effect on angiogenesis and vice versa. We saw improved vascular development along with alveolarization in ICG001-treated CTGFlungs. These results are consistent with our previous findings in hyperoxia-induced neonatal lung injury model which demonstrated that inhibition of $\beta$-catenin signaling by ICG001 improved both alveolarization and vascular development (18). We have also previously shown that CTGF is an important factor that mediates hyperoxia activation of $\beta$-catenin signaling in neonatal lung injury (6). Taken together, our findings indicate that activation of CTGF- $\beta$-catenin signaling pathway negatively regulates alveolar and vascular development during postnatal lung development and hyperoxia-induced neonatal lung injury.

To reveal the potential paracrine mechanisms by which $\beta$-catenin signaling mediates CTGF-induced lung pathology, we further examined the effects of ICG001 on pulmonary vascular remodeling. Indeed, we found that administration of ICG001 drastically decreased MWT of peripheral pulmonary vessels in CTGF transgenic mice, and this decreased MWT is correlated with decreased vascular smooth muscle cell proliferation and expression of fibronectin. These findings support that CTGF secreted by AT II cells could elicit paracrine effects on pulmonary vascular smooth muscle cells, and this is at least in part mediated by $\beta$-catenin signaling. The role of $\beta$-catenin signaling in vascular smooth muscle cell proliferation and remodeling has been revealed by numerous studies. $\beta$-Catenin signaling regulates the proliferation and differentiation of vascular smooth muscle cells during embryonic and postnatal angiogenesis, intimal thickening after vascular injury, and development of pulmonary arterial hypertension $(34,35)$. Cyclin D1 is identified as one of $\beta$-catenin targets that regulates vascular smooth muscle cell proliferation. Increased cycling D1 expression is often associated with activation of $\beta$-catenin signaling in vascular smooth muscle cells and proliferative vasculopathy such as neointima formation. We showed here that inhibition of $\beta$-catenin signaling downregulated cyclin D1 expression in CTGF transgenic lungs suggesting that cyclin D1 is one of the CTGF- $\beta$-catenin targets that may cause vascular smooth muscle cell proliferation in the neonatal lungs. Fibronectin, another $\beta$-catenin target gene, is not only a key component of vascular ECM but also an important regulator of pulmonary vascular remodeling and pulmonary arterial hypertension (36). We found that administration of ICG001 downregulated fibronectin gene expression and decreased fibronectin protein expression in peripheral pulmonary vessels in CTGF transgenic lungs. Thus, fibronectin is another CTGF$\beta$-catenin target that participates in vascular remodeling. We also showed that inhibition of $\beta$-catenin signaling downregulates CTGF-induced collagen 1al expression. Collagen 1a1 is one of the most important ECM that is associated with progressive pulmonary and vascular fibrosis (37). Many studies have demonstrated that CTGF upregulates collagen 1a1 expression in many types of cells including lung fibroblasts and vascular smooth muscle cells. This study highlights a $\beta$-catenin-dependent mechanism by which CTGF upregulates collagen 1a1 expression in the neonatal lung.

Our previous studies have demonstrated that targeted overexpression of CTGF in AT II cells induces PH in neonatal mice, and this is probably caused by decreased vascular development and increased pulmonary vascular remodeling (12). We showed here that the increased pulmonary vascularization and decreased vascular remodeling by $\beta$-catenin inhibition leads to decreased RVSP and RVH, suggesting decreased $\mathrm{PH}$. These data are consistent with our previous studies in hyperoxia-induced neonatal lung injury model that treatment with CTGF-neutralizing antibodies and ICG001decreased PH $(6,18)$. PH is often associated with severe BPD. Despite widespread availability and use of pulmonary vasodilators such as nitric oxide, the mortality rate of infants with BPD complicated by $\mathrm{PH}$ continues to remain as high as $50 \%(3,38)$. Therapeutic agents that target distinct pathogenic mechanisms of pulmonary vascular remodeling and growth in severe BPD may provide additional relief on $\mathrm{PH}$ and decrease mortality. Thus, targeting CTGF- $\beta$-catenin signaling to alleviate pulmonary vascular remodeling may be a promising new therapy for $\mathrm{PH}$ in severe BPD.

There are potential limitations of this study. First, $\beta$-catenin signaling plays an important role in regulating organ development and tissue remodeling other than the lung. ICG001 was given systemically which could have extra pulmonary effects. We did monitor animal somatic growth and survival and did not observe any difference among the study groups (data not shown). Second, it is unclear how CTGF activates $\beta$-catenin signaling in the neonatal lung. Our previous in vitro 
studies have provided evidence that overexpression of CTGF in AT II cells induces EMT via ILK, suggesting an autocrine mechanism via integrin pathway (31). We have also showed that LRP5/6 is important in hyperoxia activation of $\beta$-catenin signaling in vascular smooth muscle cells but not in alveolar epithelial cells of the neonatal lung (39). Future studies will be designed to answer the question whether CTGF differentially activates integrin and LRP5/6 in the epithelial and mesenchymal compartments of the neonatal lung. Third, it is unknown if CTGF modulates other intracellular kinases such as extracellular signal-regulated kinase, p42/44 MAPK, and Smad2/3 pathways in the neonatal lung. CTGF has been reported to be able to activate all of these transcription modulators in cell- and tissue-dependent manners. It would be important to further delineate the role of these kinases in mediating CTGF-induced lung pathology that may contribute to our overall understanding in the signaling mechanisms regulating neonatal lung development and injury repair.

In conclusion, we have demonstrated that inhibition of $\beta$-catenin transcriptional activity by ICG001 reversed CTGFinduced BPD-like pathology and $\mathrm{PH}$ in neonatal mice. We also observed that the improvement of alveolar and vascular structures is associated with downregulation of several $\beta$-catenin targets including cyclin D1, collagen 1a1, and fibronectin that regulates cell proliferation and ECM deposition. Thus, the effects of CTGF on epithelial and mesenchymal compartments in the neonatal lung are likely mediated by $\beta$-catenin-dependent autocrine and paracrine mechanisms. Therefore, targeting CTGF- $\beta$-catenin signaling may provide a novel strategy in the prevention and treatment of BPD. Given that CTGF and $\beta$-catenin are variably involved in adult lung pathology such as idiopathic pulmonary fibrosis and $\mathrm{PH}$, our study also provide a target for these adult lung diseases.

\section{METHODS}

\section{Mouse Model With Targeted Overexpression of CTGF in AT II Cells}

The study protocols were reviewed and approved by the Animal Care and Use Committee at the University of Miami School of Medicine (Miami, FL). Two lines of transgenic mice, the SP-C-rtTA mice (line 2), bearing the reverse tetracycline responsive transactivator (rtTA) under the control of the $3.7-\mathrm{kb}$ rat SP-C gene promoter, and the TetOCTGF mice, containing tetracycline operator and minimal CMV promoter and CTGF transgene were mated to produce CTGF transgenic (CTGF) and control pups as previously described (12). Induction of CTGF expression by doxycycline was started on postnatal day (P) 1 and genotyping was performed as previously described (12).

\section{Bronchoalveolar Lavage and Serum Collection}

Bronchoalveolar lavage (BAL) was performed in 4-wk-old control and CTGF mice by instilling $0.5 \mathrm{ml}$ cold normal saline into the airway through a trachea catheter and gently withdrawing the fluid, as described (12). Blood samples were collected through right ventricular puncture in 4-wk-old control and CTGF mice that are anesthetized. After allowed to clot at room temperature for $30 \mathrm{~min}$, the blood sampling tubes were centrifuged at $1,000 \mathrm{~g}$ for $10 \mathrm{~min}$, the serum was collected and stored at $-80^{\circ} \mathrm{C}$.

\section{CTGF ELISA}

CTGF was measured in BAL and serum by a competition ELISA using an affinity-purified goat polyclonal antibody to human CTGF (40). Briefly, the capture plate, a high binding flat-bottom ELISA plate (96-well; Corning Costar, Cambridge, MA) was coated with0.5 ng of $\mathrm{CTGF} /$ well. Competition mixtures of anti-CTGF with either CTGF standards, BAL $(50 \mu \mathrm{l})$, or serum samples $(50 \mu \mathrm{l})$ were prepared in triplicates in low binding round bottom plates (96 well; Sarstedt, Newton, NC). Following overnight incubations of both plates at $4^{\circ} \mathrm{C}$ and blocking of the capture plate, the competition mixtures were transferred to the capture plate and incubated at room temperature for $2 \mathrm{~h}$. Following washes the capture plate was further incubate with an alkaline phosphotase-linked rabbit antigoat IgG detection antibody (KPL, Gaithersburg, MD) for $2 \mathrm{~h}$ at room temperature. The wells were washed again and incubated with an alkaline phosphatase substrate solution (p-nitrophenyl phosphate, Sigma Chemical, St Louis, $\mathrm{MO}$ ) and then absorbances were measured at $405 \mathrm{~nm}$ on a microplate reader (BioRad, Hercules, CA). This assay has a sensitivity of $1.0 \mathrm{ng} /$ well of CTGF and an intra-assay variability of $5 \%$.

\section{ICG001 Injection and Tissue Preparation}

On P5, control and CTGF pups were randomized to four groups to receive ICG001 (dissolved in dimethyl sulfoxide) or dimethyl sulfoxide (placebo). ICG001 (10 mg/kg/dose) or placebo (equal volume) was given by i.p. injection once a day for total of $10 \mathrm{~d}$. On P15, mice were killed, and the lungs were infused with $4 \%$ paraformaldehyde via a tracheal catheter at $20 \mathrm{cmH}_{2} \mathrm{O}$ of pressure for $5 \mathrm{~min}$ and then fixed in $4 \%$ paraformaldehyde solution overnight at $4{ }^{\circ} \mathrm{C}$. Fixed lung tissues were paraffin embedded, and $5-\mu \mathrm{m}$ sections were processed. Additional lungs were also collected for total RNA and protein isolation.

\section{Lung Histology and Morphometry}

Lung tissue sections were stained by standard hematoxylin and eosin method for histology and morphometry. Mean linear intercept was measured by a staff unaware of the experimental condition as previously described (12).

\section{Assessment of $\beta$-Catenin Nuclear Translocation Index}

Immunofluorescence staining for $\beta$-catenin and DAPI nuclear staining were performed on lung sections. $\beta$-Catenin nuclear translocation index was determined by the percentage of $\beta$-catenin-positive nuclei to total nuclei (12).

\section{Pulmonary Vascular Morphometry}

To determine vascular density, immunofluorescence staining for vWF, an endothelial marker, was performed. Five random images were taken under the $\times 20$ magnification on each lung section, and the average number of vWF stained vessels $(<50 \mu \mathrm{m}$ in diameter) was calculated.

\section{Assessment of Pulmonary Vascular Remodeling}

MWT of peripheral pulmonary vessels $(<50 \mu \mathrm{m}$ in diameter $)$ was determined on $\alpha$-SMA-stained tissue sections as previously described (12). For assessing proliferation of vascular smooth muscle cells, double immunofluorescence staining for Ki67 and $\alpha$-SMA was performed. The numbers of vessels with at least one Ki67-positive nucleus in smooth muscle cells were counted from 20 vessels $(<50$ $\mu \mathrm{m}$ in diameter) on each slide. Fibronectin expression was determined by immunofluorescence staining.

\section{Assessment of $\mathrm{PH}$}

On P15, (RVSP and right ventricle (RV) to left ventricle (LV) plus septum weight ratio $(\mathrm{RV} / \mathrm{LV}+\mathrm{S})$ were determined as indices for $\mathrm{PH}$ as previously described (12).

\section{RNA Isolation and Quantitative Real-Time RT-PCR}

Total RNA was isolated from frozen lung tissues and treated with DNase to remove possible DNA contamination as described (12). One $\mu \mathrm{g}$ of total RNA was reverse transcribed. Real-time RT-PCR was performed on an ABI Fast 7500 System (Applied Biosystems, Foster City, CA). The mRNA expression levels of target genes were determined from the standard curve and normalized to $18 \mathrm{~S}$ rRNA as described (12).

\section{Western Blot Analysis}

Total protein was extracted from frozen lung tissues with a RIPA buffer according to manufacturer's protocol (Santa Cruz, Dallas, TX). Western blot analysis was performed to assess target protein 
expression in lung homogenates as previously described (12, also see this reference for antibody used).

\section{Data Presentation and Statistical Analysis}

Data were expressed as means \pm SD and analyzed by one-way ANOVA followed by Student-Newman-Keuls test. A $P<0.05$ was considered significant.

\section{STATEMENT OF FINANCIAL SUPPORT}

Financial support has been received from the Project Newborn from the University of Miami (S.W.) and Micah Batchelor Award from the Batchelor Foundation, Miami, Florida (S.W.).

Disclosure: None.

\section{REFERENCES}

1. Jobe AH, Bancalari E. Bronchopulmonary dysplasia. Am J Respir Crit Care Med 2001;163:1723-9.

2. Bhandari A, Bhandari V. Pitfalls, problems, and progress in bronchopulmonary dysplasia. Pediatrics 2009;123:1562-73.

3. Stenmark KR, Abman SH. Lung vascular development: implications for the pathogenesis of bronchopulmonary dysplasia. Annu Rev Physiol 2005;67:623-61.

4. Leask A, Abraham DJ. All in the CCN family: essential matricellular signaling modulators emerge from the bunker. J Cell Sci 2006;119:4803-10.

5. Kambas K, Chrysanthopoulou A, Kourtzelis I, et al. Endothelin-1 signaling promotes fibrosis in vitro in a bronchopulmonary dysplasia model by activating the extrinsic coagulation cascade. J Immunol 2011;186:6568-75.

6. Alapati D, Rong M, Chen S, et al. Connective tissue growth factor antibody therapy attenuates hyperoxia-induced lung injury in neonatal rats. Am J Respir Cell Mol Biol 2011;45:1169-77.

7. Alejandre-Alcázar MA, Kwapiszewska G, Reiss I, et al. Hyperoxia modulates TGF-beta/BMP signaling in a mouse model of bronchopulmonary dysplasia. Am J Physiol Lung Cell Mol Physiol 2007;292:L537-49.

8. Chen CM, Wang LF, Chou HC, Lang YD, Lai YP. Up-regulation of connective tissue growth factor in hyperoxia-induced lung fibrosis. Pediatr Res 2007;62:128-33.

9. Shimada I, Matsui K, Brinkmann B, et al. Novel transcript profiling of diffuse alveolar damage induced by hyperoxia exposure in mice: normalization by glyceraldehyde 3-phosphate dehydrogenase. Int J Legal Med 2008; 122:373-83.

10. Wu S, Capasso L, Lessa A, et al. High tidal volume ventilation up-regulates CTGF expression in the lung of newborn rats. Pediatr Res 2008;63:245-50.

11. Wu S, Platteau A, Chen S, McNamara G, Whitsett J, Bancalari E. Conditional overexpression of connective tissue growth factor disrupts postnatal lung development. Am J Respir Cell Mol Biol 2010;42:552-63.

12. Chen S, Rong M, Platteau A, et al. CTGF disrupts alveolarization and induces pulmonary hypertension in neonatal mice: implication in the pathogenesis of severe bronchopulmonary dysplasia. Am J Physiol Lung Cell Mol Physiol 2011;300:L330-40.

13. Gao R, Brigstock DR. Connective tissue growth factor (CCN2) induces adhesion of rat activated hepatic stellate cells by binding of its C-terminal domain to integrin alpha(v)beta(3) and heparan sulfate proteoglycan. J Biol Chem 2004;279:8848-55.

14. Heng EC, Huang Y, Black SA Jr, Trackman PC. CCN2, connective tissue growth factor, stimulates collagen deposition by gingival fibroblasts via module 3 and alpha6- and beta1 integrins. J Cell Biochem 2006;98:409-20.

15. Mercurio S, Latinkic B, Itasaki N, Krumlauf R, Smith JC. Connectivetissue growth factor modulates WNT signalling and interacts with the WNT receptor complex. Development 2004;131:2137-47.

16. Deng YZ, Chen PP, Wang Y, et al. Connective tissue growth factor is overexpressed in esophageal squamous cell carcinoma and promotes tumorigenicity through beta-catenin-T-cell factor/Lef signaling. J Biol Chem 2007;282:36571-81.

17. Emami KH, Nguyen $\mathrm{C}, \mathrm{Ma} \mathrm{H}$, et al. A small molecule inhibitor of betacatenin/CREB-binding protein transcription [corrected]. Proc Natl Acad Sci USA 2004;101:12682-7.

18. Alapati D, Rong M, Chen S, Hehre D, Hummler SC, Wu S. Inhibition of $\beta$-catenin signaling improves alveolarization and reduces pulmonary hypertension in experimental bronchopulmonary dysplasia. Am J Respir Cell Mol Biol 2014;51:104-13.

19. Ivkovic S, Yoon BS, Popoff SN, et al. Connective tissue growth factor coordinates chondrogenesis and angiogenesis during skeletal development. Development 2003;130:2779-91.

20. Sonnylal S, Shi-Wen X, Leoni P, et al. Selective expression of connective tissue growth factor in fibroblasts in vivo promotes systemic tissue fibrosis. Arthritis Rheum 2010;62:1523-32.

21. Sonnylal S, Xu S, Jones $\mathrm{H}$, et al. Connective tissue growth factor causes EMT-like cell fate changes in vivo and in vitro. J Cell Sci 2013;126:2164-75.

22. Clevers $\mathrm{H}$. Wnt/beta-catenin signaling in development and disease. Cell 2006;127:469-80.

23. Oloumi A, McPhee T, Dedhar S. Regulation of E-cadherin expression and beta-catenin/Tcf transcriptional activity by the integrin-linked kinase. Biochim Biophys Acta 2004;1691:1-15.

24. Dasgupta C, Sakurai R, Wang Y, et al. Hyperoxia-induced neonatal rat lung injury involves activation of TGF- $\{$ beta $\}$ and Wnt signaling and is protected by rosiglitazone. Am J Physiol Lung Cell Mol Physiol 2009;296:L1031-41.

25. Popova AP, Bentley JK, Anyanwu AC, et al. Glycogen synthase kinase$3 \beta / \beta$-catenin signaling regulates neonatal lung mesenchymal stromal cell myofibroblastic differentiation. Am J Physiol Lung Cell Mol Physiol 2012;303:L439-48

26. Shtutman M, Zhurinsky J, Simcha I, et al. The cyclin D1 gene is a target of the beta-catenin/LEF-1 pathway. Proc Natl Acad Sci USA 1999;96:5522-7.

27. Douglas IS, Diaz del Valle F, Winn RA, Voelkel NF. Beta-catenin in the fibroproliferative response to acute lung injury. Am J Respir Cell Mol Biol 2006;34:274-85.

28. Villar J, Cabrera NE, Valladares F, et al. Activation of the Wnt/ $\beta$-catenin signaling pathway by mechanical ventilation is associated with ventilatorinduced pulmonary fibrosis in healthy lungs. PLoS One 2011;6:e23914.

29. Baarsma HA, Spanjer AI, Haitsma G, et al. Activation of $\mathrm{WNT} / \beta$-catenin signaling in pulmonary fibroblasts by TGF- $\beta_{1}$ is increased in chronic obstructive pulmonary disease. PLoS One 2011;6:e25450.

30. Clarke DL, Carruthers AM, Mustelin T, Murray LA. Matrix regulation of idiopathic pulmonary fibrosis: the role of enzymes. Fibrogenesis Tissue Repair 2013;6:20.

31. Shafieian $\mathrm{M}$, Chen $\mathrm{S}$, Wu S. Integrin-linked kinase mediates CTGFinduced epithelial to mesenchymal transition in alveolar type II epithelial cells. Pediatr Res 2015;77:520-7.

32. Mucenski ML, Wert SE, Nation JM, et al. Beta-catenin is required for specification of proximal/distal cell fate during lung morphogenesis. J Biol Chem 2003;278:40231-8.

33. Mucenski ML, Nation JM, Thitoff AR, et al. Beta-catenin regulates differentiation of respiratory epithelial cells in vivo. Am J Physiol Lung Cell Mol Physiol 2005;289:L971-9.

34. Königshoff M, Eickelberg O. WNT signaling in lung disease: a failure or a regeneration signal? Am J Respir Cell Mol Biol 2010;42:21-31.

35. Cohen ED, Ihida-Stansbury K, Lu MM, Panettieri RA, Jones PL, Morrisey $\mathrm{EE}$. Wnt signaling regulates smooth muscle precursor development in the mouse lung via a tenascin C/PDGFR pathway. J Clin Invest 2009;119:2538-49.

36. Chiang HY, Korshunov VA, Serour A, Shi F, Sottile J. Fibronectin is an important regulator of flow-induced vascular remodeling. Arterioscler Thromb Vasc Biol 2009;29:1074-9.

37. Wang X, LeMaire SA, Chen L, et al. Increased collagen deposition and elevated expression of connective tissue growth factor in human thoracic aortic dissection. Circulation 2006;114:Suppl 1:I200-5.

38. Khemani E, McElhinney DB, Rhein L, et al. Pulmonary artery hypertension in formerly premature infants with bronchopulmonary dysplasia: clinical features and outcomes in the surfactant era. Pediatrics 2007;120: 1260-9.

39. Alapati D, Rong M, Chen S, Lin C, Li Y, Wu S. Inhibition of LRP5/6-mediated $\mathrm{Wnt} / \beta$-catenin signaling by Mesd attenuates hyperoxia-induced pulmonary hypertension in neonatal rats. Pediatr Res 2013;73:719-25.

40. Duncan MR, Frazier KS, Abramson S, et al. Connective tissue growth factor mediates transforming growth factor beta-induced collagen synthesis: down-regulation by cAMP. FASEB J 1999;13:1774-86. 